\title{
Existence and non-existence of positive solutions for nonlinear elliptic singular equations with natural growth
}

\author{
José Carmona ${ }^{a}$, Pedro J. Martínez-Aparicio ${ }^{\mathrm{b}}$, Antonio Suárez ${ }^{\mathrm{c}}$ \\ ${ }^{a}$ Departamento de Matemáticas, Universidad de Almería, Ctra. Sacramento s/n, La \\ Cañada de San Urbano,04120 - Almería, Spain. jcarmona@ual.es \\ ${ }^{b}$ Departamento de Matemática Aplicada y Estadística, Universidad Politécnica de \\ Cartagena,30202 - Murcia, Spain.pedroj.martinez@upct.es \\ ${ }^{c}$ Departamento de Ecuaciones Diferenciales y Análisis Numérico, Facultad de \\ Matemáticas, Calle Tarfia s/n, 41012-Sevilla, Spain.suarez@us.es
}

\begin{abstract}
In this paper we analyze the existence, non-existence and uniqueness of positive solutions of some nonlinear elliptic equations containing singular terms and natural growth in the gradient. We use an adequate sub-supersolution method to prove the existence of solutions, different arguments for the nonexistence and results from Arcoya-Segura de León for the uniqueness.
\end{abstract}

Key words: Nonlinear elliptic equations, natural growth condition, the sub-supersolution method, Singular gradient terms.

2010 MSC: 35A01, 35A02, 35B09, 35B51, 35D30, 35J60, 35J75.

\section{Introduction}

We study existence, nonexistence and uniqueness of positive solutions to the following nonlinear elliptic problem

$$
\begin{cases}-\Delta u+g(u)|\nabla u|^{2}=f(\lambda, u) & \text { in } \Omega \\ u=0 & \text { on } \partial \Omega\end{cases}
$$

where $\Omega$ is a smooth bounded domain of $\mathbb{R}^{N}(N \geq 3)$ and $\lambda$ is a real parameter. The functions $f \in C(\mathbb{R} \times[0,+\infty))$ and $g \in C(0,+\infty)$ are given, for some $k, \gamma, p, q>0$, by

$$
\begin{gathered}
g(s)=\frac{k}{s^{\gamma}}, \forall s>0 \\
f(\lambda, s)=\lambda s^{q} \quad \text { or } \quad f(\lambda, s)=\lambda s-s^{p}, \forall s>0 .
\end{gathered}
$$


We say that a solution of (1.1) is a function $u \in H_{0}^{1}(\Omega)$ such that $0<u$ almost everywhere in $\Omega, g(u)|\nabla u|^{2} \in L^{1}(\Omega), f(\lambda, u) \in L^{1}(\Omega)$ and

$$
\int_{\Omega} \nabla u \cdot \nabla \phi+\int_{\Omega} g(u)|\nabla u|^{2} \phi=\int_{\Omega} f(\lambda, u) \phi
$$

for every $\phi \in H_{0}^{1}(\Omega) \cap L^{\infty}(\Omega)$.

This kind of equations (with quadratic gradient terms) has attracted much interest since the pioneering works [8, 9]. In the last years, attention has been paid in singular terms in front of the gradient terms [2, 3, 4, 5, 6, 11. In fact, in most of these papers the right hand side of the equation is not identically zero, i.e. for different kind of nonnegative functions $g$ and $f$, is studied the equation

$$
\begin{cases}-\Delta u+g(u)|\nabla u|^{2}=f(\lambda, u)+f_{0} & \text { in } \Omega, \\ u=0 & \text { on } \partial \Omega,\end{cases}
$$

where $0 \leq f_{0} \in L^{r}(\Omega)(r \geq 1)$ and $f_{0} \not \equiv=0$. Thanks to this fact some aspects in the study of existence of solution of (1.4) can be simplified since the singularity can be avoided in compactly embedding subsets of $\Omega$.

When $f(\lambda, u) \equiv 0$ and $g$ is continuous at zero, problem (1.4) was studied, among others, in [8, 9]. This was the starting point in [4, 6, 11] for the singular case, to consider $f(\lambda, u) \equiv 0$ and $g(s)$ singular at zero, as the model $k / s^{\gamma}$. In [2] the authors showed the existence of positive solutions for $\gamma<2$ and non-existence for $\gamma \geq 2$. Moreover, in [5] it is proved that the solution is unique in the case $\gamma<1$.

When $f(\lambda, s)=\lambda s$ and $g \equiv 1$, in [1] it was proved that there exists a positive solution of (1.4) for every $\lambda>0$ showing the regularizing effect of the quadratic gradient terms. In 3 it was proved that this regularizing effect remains true while

$$
g(s) \geq k / s^{\gamma}, \quad \gamma<1, \quad s \text { large. }
$$

In particular, for $g$ given by (1.2) the existence of positive solution of (1.4) is proved for every $\lambda>0$, see also [10].

For $f(\lambda, s)=\lambda s^{q}$ and $g(s)=k / s^{\gamma}$ with $0<\gamma<1$ and $\gamma+q<2$ the existence of positive solution of (1.4) for every $\lambda>0$ was proved in [10], see also [3].

Very few is known for the problem (1.4) if $f_{0} \equiv 0$, mostly in the case of functions $g$ that are continuous at zero. In this case, it is proved in [3] the 
existence of positive solution for every $\lambda>0$ if $f(\lambda, s)=\lambda s^{q}$ with $q<1$. If $q=1$ and $g$ is a continuous function satisfying (1.5), in [3] is obtained existence of solution for $\lambda>\lambda_{1}$, where $\lambda_{1}$ denotes the first eigenvalue of $-\Delta$ under homogeneous Dirichelt boundary conditions. In [14], for $f(\lambda, s)=\lambda s^{q}$ with $q>1$ and given a continuous nonnegative function $g$ satisfying $(1.5)$, it is proved existence of positive solution if and only if $\lambda \geq \lambda^{*}$ for some $\lambda^{*}>0$. Moreover, multiplicity of solution is shown for $\lambda>\lambda^{*}$. Finally if $g \equiv 1$, the case $f(\lambda, s)=\lambda s-s^{p}$, for some $p>1$, was analyzed in 15] showing the existence and uniqueness of conti nuously differentiable positive solution for $\lambda>\lambda_{1}$.

In this paper we study problem (1.4) with $f_{0} \equiv 0$ in the case of functions $g$ that are singular at zero. More precisely, for the sake of clarity, we consider problem (1.1) with functions $g$ and $f$ given respectively by (1.2) and (1.3). We summarize the main results here. We would like to point out that the first result contains direct results but we include it for reader's convenience.

Theorem 1.1. Assume that $f(\lambda, u)=\lambda u$ and $g(s)=\frac{k}{s \gamma}$.

1. If $\gamma<1$, there exists a positive solution of (1.1) if and only if $\lambda>\lambda_{1}$. Moreover, for $\lambda>\lambda_{1}$ there exists a unique bounded positive solution.

2. If $\gamma=1$ and $k<1$, then there exists positive solution of (1.1) if and only if $\lambda=\lambda_{1} /(1-k)$. In this case, there exist infinite positive solutions.

3. If $\gamma=1$ and $k \geq 1$, then (1.1) has no positive solution for $\lambda>0$.

4. If $\gamma>1$ then (1.1) has no positive solution for $\lambda>0$.

Theorem 1.2. Assume that $f(\lambda, u)=\lambda u^{q}, 0<q<1$ and $g(s)=\frac{k}{s^{\gamma}}$.

1. If $\gamma<1$, there exists a unique bounded positive solution of (1.1) for every $\lambda>0$.

2. If $\gamma=1$ and $k \leq q$, there exists at most one positive solution for every $\lambda>0$.

3. If $\gamma+q>2$, then (1.1) has no positive solution for $\lambda>0$.

Theorem 1.3. Assume that $f(\lambda, u)=\lambda u^{q}, 1<q$ and $g(s)=\frac{k}{s^{\gamma}}$.

1. If $\gamma<1$ and $\gamma+q<2$, there exists $\lambda^{*}>0$ such that 1.1) possesses a positive solution for every $\lambda \geq \lambda^{*}$ and (1.1) does not possess any positive solution for every $\lambda<\lambda^{*}$.

2. If $\gamma \geq 1$ then (1.1) has no positive solution for $\lambda>0$. 
Theorem 1.4. Assume that $f(\lambda, u)=\lambda u-u^{p}, p>1$, and $g(s)=\frac{k}{s^{\gamma}}$.

1. Any positive solution $u$ of (1.1) is bounded and $\|u\|_{\infty} \leq \lambda^{1 /(p-1)}$.

2. If $\gamma<1$, there exists a positive solution of (1.1) if and only if $\lambda>\lambda_{1}$. In this case, the solution is unique.

3. If $\gamma=1$ and $k<1$, if there exists positive solution of (1.1) then it is unique and $\lambda>\lambda_{1} /(1-k)$.

4. If $\gamma=1$ and $k \geq 1$, then (1.1) has no positive solution for $\lambda \geq 0$.

5. If $\gamma>1$, then (1.1) has no positive solution for $\lambda \geq 0$.

To prove our existence results, we use the sub-supersolution method for weak-solution. In [9] it was proved that the sub-supersolution method works for sub and supersolution that belong to $W^{1, \infty}(\Omega)$ and $g$ is regular. We present an adequate method using the Schauder Fixed Point Theorem assuming some general conditions on the nonlinear function $f$. In our case, we can only apply this method for the case $g$ integrable, that is $\gamma<1$. In order to use the sub-supersolution method we need a slightly improvement of the comparison principle in [5]. This is only needed for the logistic nonlinearity but we present the sub-supersolution method in the more general version.

It is well-know that the uniqueness is a very hard work, in fact very few results are concerned to the uniqueness of positive solution of (1.1). We use mainly [5], and a variant of this result, to show our uniqueness results.

Finally, we employ different arguments to show the non-existence results, some of them are an adequate extension of those in [2, 3, 14. For $\gamma \geq 1$ and $\gamma+q>2$ the main novelty is that we are able to prove that any positive solution satisfies that $|\nabla u|^{2} / u^{2} \in L^{1}(\Omega)$, which is not possible as was shown in [17].

We remark that there are some gaps in our existence results, since we can not deal with the sub-supersolution method in the case $\gamma \geq 1$, but we guess that there exists solution for some $\lambda$ 's in the case $f(\lambda, s)=\lambda s^{q}$ with $\gamma \geq 1, \gamma+q<2$. Conversely, in the case $\gamma<1, \gamma+q>2$, we can not use our non existence results, but we guess that there is no solution for $\lambda>0$. Moreover, in the case $\gamma+q=2$ we only have completely described the case $\gamma=1=q$ in Theorem 1.1. The last gap relies in the uniqueness since for $\gamma=1$ and $q<1$ we only have proved the uniqueness for $k<q$.

The outline of the paper is the following, in Section 2 we prove some qualitative properties of solutions of (1.1) and in particular the nonexistence results. In Section 3 we prove that the sub-supersolution method works for 
(1.1) in the case $\gamma<1$. The uniqueness of solution is studied in Section 4 and finally in Section 5 we include the proofs of Theorems 1.1, 1.2, 1.3 and 1.4 .

Notation. As usual for every $s \in \mathbb{R}$ we consider the positive and negative parts given by $s^{+}=\max \{s, 0\}$ and $s^{-}=\min \{s, 0\}$. We denote by $T_{k}$ the usual truncature function given by $T_{k}(s)=\min \left\{k, s^{+}\right\}+\max \left\{-k, s^{-}\right\}$for every $s \in \mathbb{R}$. We denote by $|\Omega|$ the Lebesgue measure of a measurable set $\Omega$ in $\mathbb{R}^{\mathbb{N}}$. For $1 \leq p \leq+\infty,\|u\|_{p}$ is the usual norm of a function $u \in L^{p}(\Omega)$. We equipped the standard Sobolev space $H_{0}^{1}(\Omega)$ with the norm $\|u\|=\left(\int_{\Omega}|\nabla u|^{2}\right)^{1 / 2}$.

\section{Qualitative properties of solutions}

In this section we set the main properties of solutions of (1.1). More precisely, given a solution $u$, we give sufficient conditions in order to have that $u \in L^{\infty}(\Omega)$ or even that $\frac{g(u)|\nabla u|^{2}}{u^{\beta}} \in L^{1}(\Omega)$ for some $\beta>0$. This fact will be crucial to obtain our nonexistence result.

Taking into account that $g$ is nonnegative we can recover for solutions of (1.1) any of the known properties of sub-solutions of the semilinear problem

$$
\begin{cases}-\Delta u=f(\lambda, u) & \text { in } \Omega \\ u=0 & \text { on } \partial \Omega .\end{cases}
$$

In particular these properties hold for sub-solutions of (1.1) in the sense of the following definition.

Definition 2.1. A sub-solution of (1.1) is a function $\underline{u} \in H_{0}^{1}(\Omega)$ such that

1. $0<\underline{u}$ almost everywhere in $\Omega$,

2. $g(\underline{u})|\nabla \underline{u}|^{2} \in L^{1}(\Omega), f(\lambda, \underline{u}) \in L^{1}(\Omega)$,

3. for every $\phi \in H_{0}^{1}(\Omega) \cap L^{\infty}(\Omega), \phi>0$,

$$
\int_{\Omega} \nabla \underline{u} \cdot \nabla \phi+\int_{\Omega} g(\underline{u})|\nabla \underline{u}|^{2} \phi \leq \int_{\Omega} f(\lambda, \underline{u}) \phi .
$$

Similarly a super-solution of $(1.1)$ is a function $\bar{u} \in H^{1}(\Omega)$ verifying items 1 , 2 and 3 with the reverse inequality in $(2.2)$. Observe that a super-solution is allowed to be different from zero at the boundary while, since we are studying positive solutions, this is not possible for sub-solutions. 
We recall some classical results about the regularity that we can find in [16]. Concretely, the following two lemmas resume some known $L^{\infty}(\Omega)$ estimates for sub-solutions of (2.1).

Lemma 2.2. Assume that there exists $C>0$ such that $|f(\lambda, s)| \leq C\left(1+|s|^{q}\right)$ $(q<(N+2) /(N-2))$ for every $s \in \mathbb{R}(f$ subcritical $)$ and that $u$ is a subsolution of (1.1), then $u \in L^{\infty}(\Omega)$. Moreover, there exist $\alpha, \beta>0$ such that

$$
\|u\|_{\infty} \leq \alpha\|u\|^{\beta}
$$

Remark 2.3. Once we have proved that it is bounded, under conditions of the previous lemma, we have that any solution $u$ is continuous in $\Omega$ arguing as in [12] (see Remark 2.6 in [2] for a detailed proof).

Lemma 2.4. Assume that there exists $s_{0}(\lambda)$ such that $f(\lambda, s) \leq 0$ for every $s>s_{0}(\lambda)$. Assume also that $u$ is a sub-solution of (1.1) then $u \in L^{\infty}(\Omega)$ and $\|u\|_{\infty} \leq s_{0}(\lambda)$.

The following lemma will be the keystone in the proof of our nonexistence result.

Lemma 2.5. Assume that $u \in H_{0}^{1}(\Omega) \cap L^{\infty}(\Omega)$ is a sub-solution of (1.1), then $|\nabla u|^{2} / u^{\beta} \in L^{1}(\Omega)$ for every $\beta<1$. Moreover, if we assume also that there exists $\varepsilon_{0}>0$ such that for some $0<k \leq r$ and some $c_{\lambda}>0$ we have

$$
g(s) \geq \frac{k}{s}, \quad 0<s \leq \varepsilon_{0},
$$

and

$$
f(\lambda, s) \leq c_{\lambda} s^{r}, \quad 0<s \leq \varepsilon_{0},
$$

then $g(u)|\nabla u|^{2} / u^{\beta} \in L^{1}(\Omega)$ for every $\beta<k \leq r$.

Remark 2.6. Assume (2.4). Observe that $g(s)=\frac{c}{s^{\gamma}}$ satisfies (2.3) for any $k>0$ if $\gamma>1$. Thus, the conclusion of from the previous lemma for this particular function $g$ is:

1. for $\gamma>1$ we have $\frac{|\nabla u|^{2}}{u^{\sigma}} \in L^{1}(\Omega)$ for every $\sigma<\gamma+r$,

2. for $\gamma=1$ and $c \geq r$ we have $\frac{|\nabla u|^{2}}{u^{\sigma}} \in L^{1}(\Omega)$ for every $\sigma<1+r$,

3. for $\gamma=1$ and $c<r$ we have $\frac{|\nabla u|^{2}}{u^{\sigma}} \in L^{1}(\Omega)$ for every $\sigma<1+c$. 
Proof. We consider $\psi_{\varepsilon}$ given by

$$
\psi_{\varepsilon}(s)= \begin{cases}s^{1-\beta} & s>\varepsilon \\ \frac{s}{\varepsilon^{\beta}} & 0 \leq s<\varepsilon\end{cases}
$$

Taking $\psi_{\varepsilon}(u)$ as test function in $(1.1)$ we obtain

$$
\begin{gathered}
\int_{\{u>\varepsilon\}}|\nabla u|^{2} \\
\left(\frac{1-\beta}{u^{\beta}}+g(u) u^{1-\beta}\right)+\int_{\{u<\varepsilon\}}\left(g(u) \frac{u}{\varepsilon^{\beta}}+\frac{1}{\varepsilon^{\beta}}\right)|\nabla u|^{2} \\
\leq \int_{\Omega} f(\lambda, u) \psi_{\varepsilon}(u) \leq \int_{\Omega} f(\lambda, u) u^{1-\beta}<C .
\end{gathered}
$$

Therefore using Fatou Lemma in the previous inequality we obtain that $|\nabla u|^{2} / u^{\beta} \in L^{1}(\Omega)$ for every $\beta<1$. Moreover, in order to prove the second part of the lemma, we consider the function $\varphi_{\varepsilon}$ given by

$$
\varphi_{\varepsilon}(s)= \begin{cases}\frac{1}{s^{\beta}}+\beta e^{-G(s)} \int_{\varepsilon}^{s} \frac{e^{G(t)}}{t^{\beta+1}} d t & s>\varepsilon \\ \frac{s}{\varepsilon^{\beta+1}} & 0 \leq s<\varepsilon,\end{cases}
$$

and $G$ denotes any primitive of $g$. Observe that

$$
\varphi_{\varepsilon}^{\prime}(s)+g(s) \varphi_{\varepsilon}(s)=\frac{g(s)}{s^{\beta}} \text { for every } s>\varepsilon .
$$

Thus, taking $\varphi_{\varepsilon}(u)$ as test function in $(1.1)$ we obtain

$$
\int_{\{u>\varepsilon\}} \frac{g(u)}{u^{\beta}}|\nabla u|^{2}+\int_{\{u<\varepsilon\}} \frac{1}{\varepsilon^{\beta+1}}(g(u) u+1)|\nabla u|^{2} \leq \int_{\Omega} f(\lambda, u) \varphi_{\varepsilon}(u) .
$$

We claim that $\int_{\Omega} f(\lambda, u) \varphi_{\varepsilon}(u)$ is bounded as $\varepsilon$ tends to zero. Indeed, observe that 2.4 implies that for $\varepsilon<\varepsilon_{0}$ we have

$$
\int_{\{u<\varepsilon\}} f(\lambda, u) \varphi_{\varepsilon}(u) \leq c_{\lambda} \varepsilon^{r-\beta}|\Omega|
$$

and

$$
\begin{aligned}
\int_{\{u>\varepsilon\}} f(\lambda, u) \frac{1}{u^{\beta}} & =\int_{\left\{u>\varepsilon_{0}\right\}} f(\lambda, u) \frac{1}{u^{\beta}}+\int_{\left\{\varepsilon_{0}>u>\varepsilon\right\}} f(\lambda, u) \frac{1}{u^{\beta}} \\
& \leq \max _{\varepsilon_{0} \leq s \leq\|u\|_{\infty}} f(\lambda, s) \varepsilon_{0}^{-\beta}|\Omega|+c_{\lambda}\|u\|_{\infty}^{r-\beta}|\Omega| .
\end{aligned}
$$


Thus, in order to prove the claim we only have to prove that

$$
\int_{\{u>\varepsilon\}}\left(f(\lambda, u) e^{-G(u)} \int_{\varepsilon}^{u} \frac{e^{G(t)}}{t^{\beta+1}} d t\right)
$$

is bounded for $\varepsilon \rightarrow 0$. Observe that for $\varepsilon<\varepsilon_{0}$ we have

$$
\begin{aligned}
& \int_{\{u>\varepsilon\}}\left(f(\lambda, u) e^{-G(u)} \int_{\varepsilon}^{u} \frac{e^{G(t)}}{t^{\beta+1}} d t\right)=\int_{\{u>\varepsilon\}}\left(\int_{\varepsilon}^{u} \frac{f(\lambda, u)}{t^{\beta+1}} e^{-\int_{t}^{u} g(s) d s}\right) d t \\
&= \int_{\left\{u>\varepsilon_{0}\right\}}\left(\int_{\varepsilon}^{\varepsilon_{0}} \frac{f(\lambda, u)}{t^{\beta+1}} e^{-\int_{t}^{u} g(s) d s} d t+\int_{\varepsilon_{0}}^{u} \frac{f(\lambda, u)}{t^{\beta+1}} e^{-\int_{t}^{u} g(s) d s} d t\right) \\
&+\int_{\left\{\varepsilon_{0} \geq u>\varepsilon\right\}} \int_{\varepsilon}^{u} \frac{f(\lambda, u)}{t^{\beta+1}} e^{-\int_{t}^{u} g(s) d s} d t \\
& \leq \int_{\left\{u>\varepsilon_{0}\right\}} \int_{\varepsilon}^{\varepsilon_{0}} e^{-\int_{t}^{\varepsilon_{0}} \frac{k}{s} d s} \frac{1}{t^{\beta+1}} e^{-\int_{\varepsilon_{0}}^{u} g(s) d s} f(\lambda, u) d t \\
&+\max _{\varepsilon_{0} \leq s \leq\|u\|_{\infty}} f(\lambda, s) \frac{|\Omega|}{\varepsilon_{0}^{\beta+1}}+c_{\lambda} \int_{\left\{\varepsilon_{0}>u>\varepsilon\right\}} \int_{\varepsilon}^{u} e^{-\int_{t}^{u} \frac{k}{s} d s} \frac{1}{t^{\beta+1}} u^{r} d t \\
& \leq|\Omega| \max _{\varepsilon_{0} \leq s \leq\|u\|_{\infty}} f(\lambda, s)\left(\int_{0}^{\varepsilon_{0}} \frac{t^{k}}{\varepsilon_{0}^{k} t^{\beta+1}}+\frac{1}{\varepsilon_{0}^{\beta+1}}\right)+\frac{\lambda}{k-\beta} \int_{\Omega} u^{r-\beta} .
\end{aligned}
$$

Therefore using Fatou Lemma in 2.5 we obtain that $\frac{g(u)|\nabla u|^{2}}{u^{\beta}} \in L^{1}(\Omega)$ and we conclude the proof.

The following result is contained in [17] (see Theorem 1.1).

Lemma 2.7. For every $u \in H_{0}^{1}(\Omega)$ it holds that $\int_{\Omega} \frac{|\nabla u|^{2}}{u^{2}}=+\infty$. In particular, (1.1) with $g(s)$ given by (1.2) for some $\gamma \geq 2$ has no positive solution.

Using the previous two lemmas we obtain the following corollary concerning with the nonexistence of solution for the problem (1.1).

Corollary 2.8. Assume that $f$ and $g$ are given by (1.2) and (1.3).

1. If $f(\lambda, u)=\lambda u-u^{p}$ for some $p>1$ and $\gamma>1$ then problem (1.1) does not admit solution.

2. If $f(\lambda, u)=\lambda u^{q}$ for some $q>0$ with $\gamma>1$ and $\gamma+q>2$ or $\gamma=1$ and $\min \{k, q\}>1$ then problem (1.1) does not admit solution. 
Proof. Observe that it is enough to choose $\beta=2-\gamma$ in Lemma 2.5 and thus $\frac{|\nabla u|^{2}}{u^{2}} \in L^{1}(\Omega)$ which is a contradiction with Lemma 2.7 .

que todo cuadre con la idea de [5] hay que usar tambin que $f(\lambda, x, \bar{u}) \geq$ $\tilde{f}(\lambda, x, s) \geq f(\lambda, x, \underline{u})$.

Finally, we present a general result of non-existence of positive solution of a general problem using the ideas in 2. This result will be applied to show the non-existence of positive solution of (1.1) for $\lambda$ small when $f(\lambda, u)=\lambda u^{q}$ and $\gamma<1<q$.

We consider the function $g$ given by $(1.2)$ for some $\gamma<1$ and we define $G(s)$ by

$$
G(s):=\int_{0}^{s} g(t) d t \quad s>0
$$

Lemma 2.9. The function $\varphi$ given by

$$
\varphi(s)= \begin{cases}e^{-G(s)} \int_{0}^{s} e^{G(t)} d t, & s>0, \\ 0, & s=0,\end{cases}
$$

is a continuously differentiable function in $[0,+\infty)$ and it satisfies the ordinary differential equation $\varphi^{\prime}(s)+\frac{k}{s^{\gamma}} \varphi(s)=1$, for $s>0$ and $\varphi(0)=0$.

Proof. The proof is straightforward except for assuring that $\varphi$ is differentiable at zero and $\varphi^{\prime}$ is continuous at zero. In order to do that we note firstly that $\varphi$ is continuous at zero. Indeed, since $e^{G(s)}$ is nondecreasing we have $\varphi(s) \leq s$ for $s<1$. Moreover, $\frac{k}{s^{\gamma}} \varphi(s) \leq k s^{1-\gamma}$ for $s<1$, which implies that

$$
\varphi^{\prime}(0)=\lim _{s \rightarrow 0^{+}} \frac{\varphi(s)}{s}=\lim _{s \rightarrow 0} \varphi^{\prime}(s)=1 .
$$

Then $\varphi$ is differentiable at zero and $\varphi^{\prime}$ is continuous at zero.

Theorem 2.10. Assume that there exists a positive constant $C$ such that, for $\varphi$ given by (2.7),

$$
f(\lambda, s) \varphi(s) \leq \lambda C s^{2}, \quad \forall s>0 .
$$

Then the problem (1.1) has no positive solution in $H_{0}^{1}(\Omega) \cap L^{\infty}(\Omega)$ for $\lambda_{1} \geq$ $\lambda C$. 
Proof. Let $u \in H_{0}^{1}(\Omega) \cap L^{\infty}(\Omega)$ be a positive solution for (1.1) and let $\varphi \in$ $C^{1}([0,+\infty))$ given by $(2.7)$. Since $\varphi(0)=0$, we can take $v=\varphi(u)$ as test function in (1.1) to obtain

$$
\int_{\Omega}|\nabla u|^{2}\left(\varphi^{\prime}(u)+\frac{k}{u^{\gamma}} \varphi(u)\right)=\int_{\Omega} f(\lambda, u) \varphi(u) .
$$

Using now the equation satisfied by $\varphi$ we get

$$
\int_{\Omega}|\nabla u|^{2}=\int_{\Omega} f(\lambda, u) \varphi(u) \leq \lambda C \int_{\Omega} u^{2} .
$$

From 2.8 and the variational characterization of $\lambda_{1}$ we deduce that $\lambda_{1}<$ $\lambda C$.

\section{The sub-supersolution method}

In order to show the existence of positive solutions of $(1.1)$ we are going to use the sub-supersolution method for a slightly more general problem. More precisely we consider, for some $M \geq 0$ and $0 \leq h(x) \in L^{2 N /(N+2)}(\Omega)$, the following problem

$$
\begin{cases}-\Delta u+g(u)|\nabla u|^{2}+M \Psi(u) e^{G(u)}=h(x) & \text { in } \Omega, \\ u=0 & \text { on } \partial \Omega,\end{cases}
$$

where $g \in C(0,+\infty)$ is a nonnegative function which is integrable at zero, $G$ is given by (2.6) and $\Psi$ is defined by

$$
\Psi(s):=\int_{0}^{s} e^{-G(t)} d t, \quad s>0
$$

The concept of sub and super-solution for (3.1), as in Definition 2.1, is the following:

Definition 3.1. A sub-solution of $(3.1)$ is a function $\underline{u} \in H_{0}^{1}(\Omega)$ such that $0<\underline{u}$ a.e. in $\Omega, g(\underline{u})|\nabla \underline{u}|^{2}, M \Psi(\underline{u}) e^{G(\underline{u})} \in L^{1}(\Omega)$ and for every $\phi \in H_{0}^{1}(\Omega) \cap$ $L^{\infty}(\Omega), \phi>0$,

$$
\int_{\Omega} \nabla \underline{u} \cdot \nabla \phi+\int_{\Omega} g(\underline{u})|\nabla \underline{u}|^{2} \phi+M \int_{\Omega} \Psi(\underline{u}) e^{G(\underline{u})} \phi \leq \int_{\Omega} h(x) \phi .
$$


Similarly $\bar{u} \in H^{1}(\Omega)$ such that $0<\bar{u}$ a.e. in $\Omega, g(\bar{u})|\nabla \bar{u}|^{2}, M \Psi(\bar{u}) e^{G(\bar{u})} \in$ $L^{1}(\Omega)$ and for every $\phi \in H_{0}^{1}(\Omega) \cap L^{\infty}(\Omega), \phi>0$,

$$
\int_{\Omega} \nabla \bar{u} \cdot \nabla \phi+\int_{\Omega} g(\bar{u})|\nabla \bar{u}|^{2} \phi+M \int_{\Omega} \Psi(\bar{u}) e^{G(\bar{u})} \phi \geq \int_{\Omega} h(x) \phi,
$$

is called a super-solution of (3.1). We say that $u \in H_{0}^{1}(\Omega)$ is a solution of (3.1) if it is a sub and super-solution of (3.1).

The next lemma states a Comparison Principle for (3.1) similar to that contained in [5]. We include here the proof for convenience of the reader.

Lemma 3.2. Assume that $g$ is integrable at zero and $0 \leq h(x) \in L^{1}(\Omega)$. Let $\underline{u}, \bar{u}$ be a sub and a super solution of (3.1). Then $\underline{u} \leq \bar{u}$.

Proof. Following the ideas contained in Theorem 2.7 in [5] we define $\varphi_{\varepsilon}(s)=$ $\min \{\max \{\varepsilon, s\}, 1 / \varepsilon\}$, for every $s>0$ and $\varepsilon<1$. We take as test function $e^{-G\left(\varphi_{\varepsilon}(\underline{u})\right)} T_{k}\left((\Psi(\underline{u})-\Psi(\bar{u}))^{+}\right) \in H_{0}^{1}(\Omega) \cap L^{\infty}(\Omega)$ in the inequality satisfied by $\underline{u}$ and $e^{-G\left(\varphi_{\varepsilon}(\bar{u})\right)} T_{k}\left((\Psi(\underline{u})-\Psi(\bar{u}))^{+}\right) \in H_{0}^{1}(\Omega) \cap L^{\infty}(\Omega)$ in the inequality satisfied by $\bar{u}$. Subtracting and taking into account that $\Psi(s)$ is strictly increasing and $e^{-G(s)}$ is strictly decreasing we have

$$
\begin{aligned}
\int_{\Omega} e^{-G\left(\varphi_{\varepsilon}(\underline{u})\right)} & \nabla \underline{u} \cdot \nabla T_{k}\left((\Psi(\underline{u})-\Psi(\bar{u}))^{+}\right) \\
& +\int_{\{\varepsilon>\underline{u}\} \cup\{\underline{u}>1 / \varepsilon\}} e^{-G\left(\varphi_{\varepsilon}(\underline{u})\right)} g(\underline{u})|\nabla \underline{u}|^{2} T_{k}\left((\Psi(\underline{u})-\Psi(\bar{u}))^{+}\right) \\
& +M \int_{\Omega} e^{-G\left(\varphi_{\varepsilon}(\underline{u})\right)} e^{G(\underline{u})} \Psi(\underline{u}) T_{k}\left((\Psi(\underline{u})-\Psi(\bar{u}))^{+}\right) \\
& -\int_{\Omega} e^{-G\left(\varphi_{\varepsilon}(\bar{u})\right)} \nabla \bar{u} \cdot \nabla T_{k}\left((\Psi(\underline{u})-\Psi(\bar{u}))^{+}\right) \\
& -\int_{\{\varepsilon>\bar{u}\} \cup\{\bar{u}>1 / \varepsilon\}} e^{-G\left(\varphi_{\varepsilon}(\bar{u})\right)} g(\bar{u})|\nabla \bar{u}|^{2} T_{k}\left((\Psi(\underline{u})-\Psi(\bar{u}))^{+}\right) \\
& -M \int_{\Omega} e^{-G\left(\varphi_{\varepsilon}(\bar{u})\right)} e^{G(\bar{u})} \Psi(\bar{u}) T_{k}\left((\Psi(\underline{u})-\Psi(\bar{u}))^{+}\right) \\
\leq & \int_{\Omega} h(x)\left(e^{-G\left(\varphi_{\varepsilon}(\underline{u})\right)}-e^{-G\left(\varphi_{\varepsilon}(\bar{u})\right)}\right) T_{k}\left((\Psi(\underline{u})-\Psi(\bar{u}))^{+}\right) \leq 0 .
\end{aligned}
$$

Observe that functions $e^{-G\left(\varphi_{\varepsilon}(\underline{u})\right)}$ and $e^{-G\left(\varphi_{\varepsilon}(\bar{u})\right)}$ are bounded and thus we can 
pass to the limit as $\varepsilon$ goes to zero and we obtain that

$$
\begin{aligned}
0 & \leq \int_{\Omega}\left|\nabla T_{k}\left((\Psi(\underline{u})-\Psi(\bar{u}))^{+}\right)\right|^{2} \\
& +M \int_{\Omega}(\Psi(\underline{u})-\Psi(\bar{u})) T_{k}\left((\Psi(\underline{u})-\Psi(\bar{u}))^{+}\right) \leq 0 .
\end{aligned}
$$

Thus $(\Psi(\underline{u})-\Psi(\bar{u}))^{+}=0$ and consequently $\underline{u} \leq \bar{u}$ (since $\psi$ is strictly increasing).

In the next lemma we give sufficient conditions to prove the existence of solution of $(3.1)$.

Lemma 3.3 (Existence result). Let $0 \leq h(x) \in L^{2 N /(N+2)}(\Omega), h \not \equiv 0$, and assume that $g$ is integrable at zero. Then there exists a solution $u \in H_{0}^{1}(\Omega)$ of (3.1). Moreover, if $h(x) \in L^{q}(\Omega)$ for some $q>N / 2$ then $u \in L^{\infty}(\Omega)$.

Proof. We consider the approximated problem

$$
\begin{cases}-\Delta u_{n}+\frac{g_{n}\left(u_{n}\right)\left|\nabla u_{n}\right|^{2}}{1+\frac{1}{n}\left|\nabla u_{n}\right|^{2}}+\frac{M \Psi_{n}\left(u_{n}^{+}\right) e^{G_{n}\left(u_{n}^{+}\right)}}{1+\frac{1}{n} \Psi_{n}\left(u_{n}^{+}\right) e^{G_{n}\left(u_{n}^{+}\right)}}=T_{n}(h(x)) & \text { in } \Omega \\ u_{n}=0 & \text { on } \partial \Omega\end{cases}
$$

where the functions $g_{n}, G_{n}$ and $\Psi_{n}$ are given by

$$
g_{n}(t)=\frac{g\left(t^{+}+\frac{1}{n}\right)}{1+\frac{1}{n} g\left(t^{+}+\frac{1}{n}\right)} \frac{t^{+}}{\frac{1}{n}+t^{+}},
$$

$G_{n}(t)=\int_{0}^{t} g_{n}(s) d s$ and $\Psi_{n}(t):=\int_{0}^{t} e^{-G_{n}(s)} d s$, for every $t \in \mathbb{R}$.

By applying [13] there exists a solution $u_{n} \in H_{0}^{1}(\Omega)$ of (3.3) such that $u_{n} \in L^{\infty}(\Omega)$ (see [16]). Moreover we claim that $u_{n} \geq 0$. Indeed, taking $u_{n}^{-}$ as test function in 3.3 we obtain

$$
\begin{array}{r}
\int_{\Omega}\left|\nabla u_{n}^{-}\right|^{2}+\int_{\Omega} \frac{g_{n}\left(u_{n}\right) u_{n}^{-}\left|\nabla u_{n}\right|^{2}}{1+\frac{1}{n}\left|\nabla u_{n}\right|^{2}}+M \int_{\Omega} u_{n}^{-} \frac{\Psi_{n}\left(u_{n}^{+}\right) e^{G_{n}\left(u_{n}^{+}\right)}}{1+\frac{1}{n} \Psi_{n}\left(u_{n}^{+}\right) e^{G_{n}\left(u_{n}^{+}\right)}} \\
=\int_{\Omega} T_{n}(h(x)) u_{n}^{-}
\end{array}
$$

and thanks to the positivity of the lower order terms we have

$$
\int_{\Omega}\left|\nabla u_{n}^{-}\right|^{2} \leq \int_{\Omega} T_{n}(h(x)) u_{n}^{-} \leq 0
$$


which establishes that $u_{n} \geq 0$. Similarly, taking $u_{n}$ as test function in (3.3) and using the positivity of the lower order terms we get

$$
\int_{\Omega}\left|\nabla u_{n}\right|^{2} \leq \int_{\Omega} h(x) u_{n} \leq\|h\|_{2 N /(N+2)}\left\|u_{n}\right\|_{2^{*}}
$$

Thus, using the Sobolev embedding theorem, we deduce that $u_{n}$ is bounded in $H_{0}^{1}(\Omega)$. Even more, taking $T_{\varepsilon}\left(u_{n}\right) / \varepsilon$ as test function and using Fatou Lemma as $\varepsilon \rightarrow 0$ yields that

$$
\int_{\Omega}\left(g_{n}\left(u_{n}\right) \frac{\left|\nabla u_{n}\right|^{2}}{1+\frac{1}{n}\left|\nabla u_{n}\right|^{2}}+M \frac{\Psi_{n}\left(u_{n}^{+}\right) e^{G_{n}\left(u_{n}^{+}\right)}}{1+\frac{1}{n} \Psi_{n}\left(u_{n}^{+}\right) e^{G_{n}\left(u_{n}^{+}\right)}}\right) \leq\|h\|_{1} .
$$

Therefore $u_{n}$ weakly converges to $u \in H_{0}^{1}(\Omega), \nabla u_{n} \rightarrow \nabla u$ a.e. (see [7, Theorem 2.1]) and using Fatou Lemma as $n \rightarrow \infty$,

$$
g(u)|\nabla u|^{2} \chi_{\{u>0\}} \in L^{1}(\Omega) \text { and } \Psi(u) e^{G(u)} \chi_{\{u>0\}} \in L^{1}(\Omega) .
$$

In particular, since $g$ is integrable at zero, we have that $\Psi(u) e^{G(u)}$ is bounded at zero and thus, $\Psi(u) e^{G(u)} \in L^{1}(\Omega)$.

In order to pass to the limit and to prove that $u$ is the solution of (3.1) it is essential to prove that $u>0$.

In order to do that we follow the ideas in [6]. We take $e^{-G_{n}\left(u_{n}\right)} \phi$, with $0 \leq \phi \in C_{0}^{\infty}(\Omega)$, as test function in (3.3) and we obtain that

$$
\begin{aligned}
\int_{\Omega} \nabla \Psi_{n}\left(u_{n}\right) \cdot \nabla \phi+M \int_{\Omega} \Psi_{n}\left(u_{n}\right) \phi & \geq \int_{\Omega} T_{n}(h(x)) e^{-G_{n}\left(u_{n}\right)} \phi \\
& \geq \int_{\Omega} T_{1}(h(x)) e^{-G_{n}\left(u_{n}\right)} \phi .
\end{aligned}
$$

Observe that, since $e^{-G_{n}\left(u_{n}\right)}$ is bounded and $\Psi_{n}(s) \leq s$ we can pass to the limit in the inequality above and we have

$$
\int_{\Omega} \nabla \Psi(u) \cdot \nabla \phi+M \int_{\Omega} \Psi(u) \phi \geq \int_{\Omega} T_{1}(h(x)) e^{-G(u)} \phi .
$$

Thus, the strong maximum principle allows us to assure that $0<\Psi(u) \leq u$. Now, we can use the ideas in [6] to pass to the limit in the approximated problem and we deduce that $u \in H_{0}^{1}(\Omega)$ is a solution of 3.1 . The second part follows directly from Lemma 2.2 . 
As a consequence of Lemma 3.2 we state:

Corollary 3.4. The solution provided by Lemma 3.3 is unique.

Now, we are ready to state the method of sub and super solutions in order to get existence of solution of (1.1). We define the interval (closed and convex in $\left.L^{2}(\Omega)\right)$

$$
I:=[\underline{u}, \bar{u}]=\left\{u \in L^{2}(\Omega): \underline{u} \leq u \leq \bar{u}\right\} .
$$

Theorem 3.5. Assume that there exist $0<\underline{u} \leq \bar{u}$ in $\Omega$, respectively a sub and a super-solution of (1.1). Assume also that $g$ is integrable at zero and the following conditions on $f$ :

(F1) There exists a constant $M \geq 0$ such that the map $s \mapsto f(\lambda, s)+$ $M \Psi(s) e^{G(s)}$ is increasing for $s>0$.

(F2) $f(\lambda, \bar{u})+M \Psi(\bar{u}) e^{G(\bar{u})} \in L^{2 N /(N+2)}(\Omega)$.

(F3) $f(\lambda, \underline{u})+M \Psi(\underline{u}) e^{G(\underline{u})}>0$.

Then, there exists a solution $u$ of (1.1) such that $\underline{u} \leq u \leq \bar{u}$.

Proof. We define the operator $T: I \mapsto H_{0}^{1}(\Omega), w \mapsto u:=T(w)$ the unique solution of

$$
\begin{cases}-\Delta u+g(u)|\nabla u|^{2}+M \Psi(u) e^{G(u)}=F(x) & \text { in } \Omega, \\ u=0 & \text { on } \partial \Omega,\end{cases}
$$

where

$$
F(x):=f(\lambda, w(x))+M \Psi(w(x)) e^{G(w(x))} .
$$

Thanks to (F1) and (F3) we have that $F>0$. Moreover, (F1) and (F2) imply that $F \in L^{2 N /(N+2)}(\Omega)$. Thus, by Lemma 3.3 and Corollary 3.4 it follows that $T$ is well-defined. We claim now that $T$ is compact. Indeed, consider any sequence $w_{n} \in I$, weakly convergent to $w \in I$ in $L^{2}(\Omega)$. Taking $u_{n}=T\left(w_{n}\right)$, it is clear that there exists $C>0$ such that

$$
\int_{\Omega}\left|\nabla u_{n}\right|^{2} \leq C
$$

i.e. $u_{n}$ is bounded in $H_{0}^{1}(\Omega)$. Therefore there exists $u \in H_{0}^{1}(\Omega)$ such that, up to a subsequence, $u_{n} \rightarrow u$ weakly in $H_{0}^{1}(\Omega), u_{n} \rightarrow u$ strongly in $L^{\eta}(\Omega)$ with $\eta<2 N /(N-2)$ and $u_{n}(x) \rightarrow u(x)$ a.e. in $\Omega$. We can pass to the limit using 
the previous property, that $w_{n} \in I$ is bounded in $L^{2}(\Omega)$ and that $u>0$ (we can prove it using (F3) as in Lemma 3.3). Hence, $u=T(w)$. Moreover, as in [2], we can prove that $u_{n}$ strongly converges to $u$ in $H_{\text {loc }}^{1}(\Omega)$. Thus, arguing as in [3] we conclude that $u_{n}$ strongly converges to $u$ in $H_{0}^{1}(\Omega)$ and therefore $T$ is compact.

Now, we claim that $T(I) \subset I$. Indeed, take $w \in I$ and consider $u$ the unique solution of (3.4). We are going to show that $u \in I$. Observe that

$$
\begin{aligned}
-\Delta \bar{u}+g(\bar{u})|\nabla \bar{u}|^{2}+M \Psi(\bar{u}) e^{G(\bar{u})} & \geq f(\lambda, \bar{u})+M \Psi(\bar{u}) e^{G(\bar{u})} \\
& \geq f(\lambda, w)+M \Psi(w) e^{G(w)} \\
& =-\Delta u+g(u)|\nabla u|^{2}+M \Psi(u) e^{G(u)} .
\end{aligned}
$$

Then, since (F2) implies that $\psi(\bar{u}) e^{G(\bar{u})} \in L^{1}(\Omega), \bar{u}$ is a supersolution of

$$
-\Delta u+g(u)|\nabla u|^{2}+M \Psi(u) e^{G(u)}=F(x) .
$$

Hence, by Lemma 3.2 we conclude that $\bar{u} \geq u$. Similarly, it can be shown that $\underline{u} \leq u$ and the result concludes by using the Schauder Fixed Point Theorem.

Remark 3.6. Some remarks are in order concerning to (F1)-(F3). Condition $(F 1)$ is similar to the used in the classical sub-supersolution method without gradient term. (F2) is necessary to auxiliar problem (3.4) is well-posed and follows directly if the function $f(\lambda, \cdot)+M \Psi(\cdot) e^{-G(\cdot)}$ has a subcritical growth. Observe that, if $f$ is a $C^{1}$-function and $\bar{u} \in L^{\infty}(\Omega)$ then (F1) and (F2) are verified. Finally, (F3) is used to find positive solutions avoiding the singularity, which it suffices that $\underline{u}>0$.

\section{Uniqueness results}

In this section we prove our uniqueness results for problem (1.1). The first result is an immediate consequence of Theorem 3.1 in [5].

Lemma 4.1. Assume that $g(s)=k / s^{\gamma}$ with $\gamma<1$ and $f(\lambda, u)=\lambda u^{q}$ with $0<q \leq 1$. Then we can assure uniqueness of bounded solution of 1.1 .

Proof. Using Theorem 3.1 in [5], if

$$
s \mapsto h(s):=\frac{f(\lambda, s) e^{-G(s)}}{\Psi(s)} \quad \text { is decreasing }
$$


then, there exists at most a positive bounded solution of (1.1). The functions $G$ and $\Psi$ are defined in (2.6) and (3.2).

In this case, the function $h(s)$ defined in 4.1 is decreasing if

$$
j(s):=s^{q} e^{-G(s)}+\Psi(s)\left(k s^{q-\gamma}-q s^{q-1}\right)>0, \quad s>0 .
$$

Observe that if $k s^{q-\gamma}-q s^{q-1} \geq 0$, or equivalently $s^{1-\gamma} \geq q / k$ we have that $j(s)>0$. So, assume that $s<s_{0}$ where

$$
s_{0}=\left(\frac{q}{k}\right)^{1 /(1-\gamma)} .
$$

Observe that, since $\lim _{s \rightarrow 0} \Psi(s) / s=1$, we have that $j(0)=0$. Moreover,

$$
j^{\prime}(s)=\Psi(s) s^{q-2}\left(k(q-\gamma) s^{1-\gamma}+q(1-q)\right), \quad s>0 .
$$

It is clear that if $q \geq \gamma$ then $j^{\prime}(s)>0$ and so $j(s)>0$ for every $s>0$. Assume then that $q<\gamma$, which implies that $\lim _{s \rightarrow 0} j^{\prime}(s)=+\infty$. Even more $j^{\prime}(s)=0$ if and only if $s^{1-\gamma}=q(1-q) /(k(\gamma-q))$. Finally, observe that

$$
j^{\prime}\left(s_{0}\right)=\Psi\left(s_{0}\right) s_{0}^{q-2} q(1-\gamma)>0,
$$

and hence we conclude that $j(s)>0$ for every $s<s_{0}$.

A slightly improvement of Theorem 3.1 in [5] allows us to consider the case of functions $g$ that are not integrable at zero. In this case we take $G$ any primitive of $g$, for example $G(s)=\int_{1}^{s} g(t) d t$.

Theorem 4.2. Assume that $f$ and $g$ are continuous functions, $e^{-G(s)}$ is integrable at zero, the function $f(\lambda, s) e^{-G(s)}$ is bounded at zero and that

$$
\frac{f(\lambda, s) e^{-G(s)}}{\Psi(s)} \text { is a decreasing function. }
$$

Then there exists at most a solution of (1.1) in the class

$$
\mathcal{H}=\left\{u \in H_{0}^{1}(\Omega) \cap L^{\infty}(\Omega): e^{-G(u)}|\nabla u| \in L^{2}(\Omega)\right\} .
$$

Proof. We observe that, given $\phi \in C_{0}^{1}(\Omega)$ and $u \in \mathcal{H}$ a solution of (1.1), we can take $e^{-G(u)} \phi$ as test function, beacuse $u$ is continuous (see Remark 2.3), and we obtain

$$
\int_{\Omega} e^{-G(u)} \nabla u \cdot \nabla \phi=\int_{\Omega} f(\lambda, u) e^{-G(u)} \phi
$$


Using that $f(\lambda, u) e^{-G(u)} \in L^{\infty}(\Omega), e^{-G(u)}|\nabla u| \in L^{2}(\Omega)$ and the density of $C_{0}^{1}(\Omega)$ in $H_{0}^{1}(\Omega)$ we yield

$$
\int_{\Omega} \nabla \Psi(u) \cdot \nabla \phi=\int_{\Omega} f(\lambda, u) e^{-G(u)} \phi
$$

for every $\phi \in H_{0}^{1}(\Omega)$. The proof follows now exactly as in [5, Theorem $3.1]$.

Theorem 4.3. Assume that $g(s)=k / s$ for $0<k<1$.

1. In the case $f(\lambda, s)=\lambda s^{q}$ with $k \leq q<1$ there exists at most one positive solution of (1.1).

2. For $f(\lambda, s)=\lambda s-s^{p}$ with $p>1$ there exists at most one positive solution of 1.1 .

Proof. Observe that $e^{-G(s)}=s^{-k}$ and $\Psi(s)=\frac{s^{1-k}}{1-k}$. Thus, thanks to Lemma 2.5 (with $\beta=2 k-1<k$ ) we have that if $u$ is solution of (1.1) then $u \in \mathcal{H}$. Moreover, in the case of item (1), $f(\lambda, s) e^{-G(s)}=\lambda s^{q-k}$ is bounded at zero and $\frac{f(\lambda, s) e^{-G(s)}}{\Psi(s)}=\lambda(1-k) s^{q-1}$ is a decreasing function and the result follows directly from Theorem 4.2 .

In the case of item $(2), f(\lambda, s) e^{-G(s)}=\lambda s^{1-k}-s^{p-k}$ is bounded at zero and $\frac{f(\lambda, s) e^{-G(s)}}{\Psi(s)}=\lambda(1-k)-(1-k) s^{p-1}$ is a decreasing function. Thus the result follows again from Theorem 4.2 .

\section{Proof of Theorem 1.1, Theorem 1.2, Theorem 1.3 and Theo- rem 1.4}

In this section we prove the main results concerning with the model problem.

Proof of Theorem 1.1. We are going to apply Theorem 3.5 in order to deal with the existence result of item (1). Observe that (F1) is verified clearly because $f$ is increasing.

We claim that $\underline{u}:=\varepsilon \phi_{1}^{a}$ is the required sub-solution for some convenient positive constants $\varepsilon, a>0$ to be chosen later. Indeed, observe that

$$
g(\underline{u})|\nabla \underline{u}|^{2}=k a^{2}\left|\nabla \phi_{1}\right|^{2} \varepsilon^{2-\gamma} \phi_{1}^{a(2-\gamma)-2},
$$


thus, using that $\frac{\left|\nabla \phi_{1}\right|^{2}}{\phi_{1}^{\alpha}} \in L^{1}(\Omega)$ for every $\alpha<1$, we conclude that $g(\underline{u})|\nabla \underline{u}|^{2} \in$ $L^{1}(\Omega)$ if $a>1 /(2-\gamma)$. Secondly, after a calculation, we obtain:

$$
-\Delta \underline{u}+g(\underline{u})|\nabla \underline{u}|^{2}=\varepsilon a\left|\nabla \phi_{1}\right|^{2} \phi_{1}^{a-2}\left(1-a+a k \varepsilon^{1-\gamma} \phi_{1}^{a(1-\gamma)}\right)+\varepsilon \lambda_{1} a \phi_{1}^{a} .
$$

Therefore $\underline{u}$ is a sub-solution if

$$
\varepsilon a\left|\nabla \phi_{1}\right|^{2} \phi_{1}^{a-2}\left(1-a+a k \varepsilon^{1-\gamma} \phi_{1}^{a(1-\gamma)}\right)+\varepsilon \lambda_{1} a \phi_{1}^{a}-f\left(\lambda, \varepsilon \phi_{1}^{a}\right) \leq 0 .
$$

In the case $\gamma<1$ and $f(\lambda, u)=\lambda u$, the previous inequality is equivalent to

$$
a\left|\nabla \phi_{1}\right|^{2} \phi_{1}^{-2}\left(1-a+a k \varepsilon^{1-\gamma} \phi_{1}^{a(1-\gamma)}\right)<\lambda-\lambda_{1} a .
$$

Thus, taking $\frac{\lambda}{\lambda_{1}}>a>1>\frac{1}{2-\gamma}$ and $0<\varepsilon$ small, we have that $\underline{u}=\varepsilon \phi_{1}^{a}$ is a sub-solution of 1.1 .

We build now the supersolution. Consider a $C^{2}(\bar{\Omega})$ function $e \geq e_{0}>0$ in $\Omega$, such that $|\nabla e|^{2} \geq c_{0}>0$ in $\Omega$. Then, $\bar{u}:=L e$ is a supersolution if

$$
k \frac{L^{1-\gamma}}{e^{\gamma}}|\nabla e|^{2} \geq \lambda e+\Delta e .
$$

Thus, taking $L$ large enough we have that $L e$ is a super solution and

$$
\underline{u}=\varepsilon \phi_{1}^{a} \leq L e=\bar{u} .
$$

Finally, it is clear that (F2) and (F3) are verified, and the existence of positive solution for $\lambda>\lambda_{1}$ follows. Taking $\phi_{1}$ as test function we deduce that for $\lambda \leq \lambda_{1}$ does not exist any solution. The uniqueness follows directly from Lemma 4.1.

With respect to item (2) we observe that for $g(s)=k / s$ with $k<1, R \phi_{1}^{a}$ is solution of (1.1) for any $R>0$ if

$$
a\left|\nabla \phi_{1}\right|^{2} \phi_{1}^{a-2}(1-a+k a)=\left(\lambda-\lambda_{1} a\right) \phi_{1}^{a} .
$$

Taking $a=1 /(1-k)$, then the above equality holds for $\lambda=\lambda_{1} /(1-k)$.

Moreover, given a solution $u \in H_{0}^{1}(\Omega)$ of (1.1) with $g(s)=k / s, k<1$, and $f(\lambda, s)=\lambda s$ then, using Lemma 2.2 and Lemma 2.5 (observe that $2 k<k+1$ ), the function $w:=\int_{0}^{u} e^{-G(t)} d t=\frac{u^{1-k}}{1-k}$ belongs to $H_{0}^{1}(\Omega)$. Even more, for $\phi_{n} \rightarrow \phi_{1}$ with $\phi_{n} \in C_{0}^{1}(\Omega)$ we can take $e^{-G(u)} \phi_{n}=\frac{\phi_{n}}{u^{k}}$ as test function and it follows that

$$
\int_{\Omega} \nabla w \cdot \nabla \phi_{n}=\lambda(1-k) \int_{\Omega} w \phi_{n}
$$


Passing to the limit as $n$ goes to infinity we get

$$
\lambda_{1} \int_{\Omega} w \phi_{1}=\lambda(1-k) \int_{\Omega} w \phi_{1} .
$$

Then we obtain that $\lambda_{1}=\lambda(1-k)$.

Item (3) is deduced similarly. Indeed if, for some $\lambda>0$ and some $k \geq 1$, there exists a solution $u \in H_{0}^{1}(\Omega)$ of (1.1) with $g(s)=k / s$ and $f(\lambda, s)=\lambda s$ then, using Lemma 2.2 and Lemma 2.5, the function $w=\frac{u^{1-c}}{1-c}$ belongs to $H_{0}^{1}(\Omega)$ for every $c<1$. Even more, for $\phi_{n} \rightarrow \phi_{1}$ with $\phi_{n} \in C_{0}^{1}(\Omega)$ we can take $\frac{\phi_{n}}{u^{c}}$ as test function and it follows that

$$
\int_{\Omega} \nabla w \cdot \nabla \phi_{n}+(k-c) \int_{\Omega} \frac{|\nabla u|^{2}}{u^{1+c}} \phi_{n}=\lambda(1-c) \int_{\Omega} w \phi_{n} .
$$

Passing to the limit as $n$ goes to infinity we get

$$
\lambda_{1} \int_{\Omega} w \phi_{1} \leq \lambda(1-c) \int_{\Omega} w \phi_{1}
$$

for every $c<1$. This is a contradiction since implies, in particular, that $\lambda_{1} \leq 0$.

Finally item (4) follows from Corollary 2.8.

Proof of Theorem 1.2. First we deal with item (1). Again, we will apply Theorem 3.5. As in the previous proof, condition (F1) is clearly verified, and we can take $\underline{u}=\varepsilon \phi_{1}^{a}$ as subsolution if

$$
\varepsilon^{1-q} a\left|\nabla \phi_{1}\right|^{2} \phi_{1}^{a(1-q)-2}\left(1-a+a k \varepsilon^{1-\gamma} \phi_{1}^{a(1-\gamma)}\right)+\lambda_{1} a \varepsilon^{1-q} \phi_{1}^{a(1-q)}<\lambda .
$$

Taking $\lambda>0$ and $\varepsilon$ small, 5.1 is verified for some $a>\frac{1}{2-\gamma}$.

As supersolution, we take the unique positive solution $w \in H_{0}^{1}(\Omega)$ of

$$
-\Delta w=\lambda w^{q}
$$

Moreover, we can choose $\varepsilon>0$ small enough to have $\underline{u} \leq w$. Again, (FQ) and (F3) are verified. The uniqueness follows from Lemma 4.1 .

With respect to item (2) it is deduced from Theorem 4.3.

Finally item (3) follows from Corollary 2.8 since $\gamma>2-q>1$. 
Proof of Theorem 1.3. Observe that item (2) follows by Corollary 2.8. We have to prove item (1). In order to do that we define the set

$$
\Lambda=\{\lambda>0: 1.1 \text { admits positive solution }\} .
$$

First we prove that $\Lambda$ is nonempty since there exists solution for $\lambda$ large enough. Once again $\underline{u}=\varepsilon \phi_{1}^{a}$ is subsolution if (5.1) is satisfied. Fix $\varepsilon>0$ such that $(1-a)+a k \varepsilon^{1-\gamma} \phi_{1}^{a(1-\gamma)}<\frac{1-a}{2}$. Thus, it is enough to have

$$
\varepsilon^{1-q} a \frac{1-a}{2}\left|\nabla \phi_{1}\right|^{2} \phi_{1}^{a(1-q)-2}+\varepsilon^{1-q} \lambda_{1} a \phi_{1}^{a(1-q)}<\lambda .
$$

Observe that there exits $\Omega_{1}$ a neighborhood of $\partial \Omega$ such that

$$
\varepsilon^{1-q} a \frac{1-a}{2}\left|\nabla \phi_{1}(x)\right|^{2} \phi_{1}(x)^{a(1-q)-2}+\varepsilon^{1-q} \lambda_{1} a \phi_{1}(x)^{a(1-q)} \leq 0, \quad x \in \Omega_{1} .
$$

Indeed, it is enough to take $\Omega_{1}$ such that

$$
\frac{2 \lambda_{1} \phi_{1}^{2}(x)}{(a-1)\left|\nabla \phi_{1}(x)\right|^{2}} \leq 1, \quad x \in \Omega_{1} .
$$

Thus (5.1) is true for every $\lambda$ such that

$$
\lambda>\sup _{x \in \Omega \backslash \Omega_{1}}\left\{\varepsilon^{1-q} a \frac{1-a}{2}\left|\nabla \phi_{1}\right|^{2} \phi_{1}^{a(1-q)-2}+\varepsilon^{1-q} \lambda_{1} a \phi_{1}^{a(1-q)}\right\} .
$$

As supersolution, take again $\bar{u}:=L e$ where $e$ is a $C^{2}(\bar{\Omega})$ function $e \geq$ $e_{0}>0$ in $\Omega$, such that $|\nabla e|^{2} \geq c_{0}>0$ in $\Omega$. Then, $\bar{u}$ is a supersolution if

$$
k \frac{L^{2-q-\gamma}}{e^{\gamma}}|\nabla e|^{2} \geq \lambda e^{q}+L^{1-q} \Delta e .
$$

Thus, taking $L$ large enough we have that $L e$ is a super solution and $\underline{u}=$ $\varepsilon \phi_{1}^{a} \leq L e=\bar{u}$. Hence, using Theorem 3.5 we conclude the existence of solution of (1.1) for $\lambda$ large. In particular, $\Lambda \neq \emptyset$.

We prove now that $\Lambda$ is an unbounded interval. Indeed, if $\mu \in \Lambda$ then for $\lambda=\mu$ problem (1.1) admits a positive solution $u_{\mu} \in H_{0}^{1}(\Omega) \cap L^{\infty}(\Omega)$. It is clear that for every $\lambda>\mu$ there exists a $L_{\lambda}$ large enough such that the pair $(\underline{u}, \bar{u})=\left(u_{\mu}, L_{\lambda} e\right)$ is a sub-supersolution of (1.1). Thus we conclude, using Theorem 3.5, that there exists a solution $u_{\lambda} \geq u_{\mu}$ and $(\mu,+\infty) \subset \Lambda$. 
Now, we prove that $\inf \Lambda=\lambda^{*}>0$, that is, 1.1 does not admit positive solution for $\lambda$ small. For that, we consider the function $\varphi$ given by (2.7) and apply Theorem 2.10. First, we claim that

$$
\frac{s^{q} \varphi(s)}{s^{2}} \leq C \text { for some } C>0 \text { and for all } s>0 .
$$

Observe that $1<q<2-\gamma<2$ and thus

$$
\lim _{s \rightarrow 0^{+}} \frac{s^{q} \varphi(s)}{s^{2}}=\lim _{s \rightarrow 0^{+}} \frac{\int_{0}^{s} e^{G(t)} d t}{e^{G(s)} s^{2-q}}=\lim _{s \rightarrow 0^{+}} \frac{1}{s^{1-q}\left(k s^{1-\gamma}+(2-q)\right)}=0 .
$$

Analogously,

$$
\lim _{s \rightarrow+\infty} \frac{s^{q} \varphi(s)}{s^{2}}=\lim _{s \rightarrow 0^{+}} \frac{1}{k s^{2-q-\gamma}+(2-q) s^{1-q}}=0 .
$$

Hence, there exists $C>0$ such that

$$
\frac{f(\lambda, s) \varphi(s)}{s^{2}}=\lambda \frac{s^{q} \varphi(s)}{s^{2}} \leq \lambda C,
$$

and so applying Theorem 2.10, (1.1) does not admit positive solution for $\lambda C \leq \lambda_{1}$, that is, for $\lambda$ small.

Now we prove that $\lambda^{*} \in \Lambda$. Indeed, we can take a strictly decreasing sequence $\lambda_{n} \rightarrow \lambda^{*}$ and $u_{n}$ the decreasing sequence of solutions of 1.1 for $\lambda=\lambda_{n}$. Since $u_{n}$ is bounded in $L^{\infty}(\Omega)$ and thus is bounded in $H_{0}^{1}(\Omega)$ then, up to a subsequence, $u_{n}$ weakly converges to $u^{*} \in H_{0}^{1}(\Omega) \cap L^{\infty}(\Omega)$. Once we prove that $u^{*} \neq 0$ we can argue as in Lemma 3.3 to prove that $u^{*}$ is a solution of (1.1) for $\lambda=\lambda^{*}$. In order to prove that $u^{*} \neq 0$ we denote by $z_{n}$ the sequence $u_{n} /\left\|u_{n}\right\|$ and observe that:

$$
1+k \int_{\Omega}\left|\nabla z_{n}\right| u_{n}^{1-\gamma}=\lambda_{n} \int_{\Omega} z_{n}^{2} u_{n}^{q-1} .
$$

In particular, if $u^{*}=0$, taking into account that $z_{n}$ is bounded in $H_{0}^{1}(\Omega), u_{n}$ bounded in $L^{\infty}(\Omega)$ and using Lebesgue Theorem to take limits in the previous identity, we reach a contradiction. Therefore (1.1) admits a solution for $\lambda^{*}$. 
Proof of Theorem 1.4. Item (1) follows directly from Lemma 2.4. We prove now the existence of item (2). Observe that for $M>0$ large, $(F 1)$ is verified taking $\bar{u}=\theta_{\lambda}$ the unique positive solution

$$
-\Delta u=\lambda u-u^{p}
$$

which exists and it is positive for $\lambda>\lambda_{1}$. Again, in this case $\underline{u}=\varepsilon \phi_{1}^{a}$ is subsolution arguing as in Theorem 1.1 .

We show now the uniqueness. In this case, the function $h(s)$ defined in (4.1) is decreasing if

$$
j(s):=\left(\lambda s-s^{p}\right) e^{-G(s)}+\left[-\lambda+p s^{p-1}+k \lambda s^{1-\gamma}-k s^{p-\gamma}\right] \Psi(s)>0, \quad s>0 .
$$

It is clear again that $j(0)=0$ and

$$
j^{\prime}(s)=\Psi(s) s^{-\gamma}\left[p(p-1) s^{p+\gamma-2}+\lambda k(1-\gamma)-k(p-\gamma) s^{p-1}\right], \quad s>0 .
$$

Since $p-1>p+\gamma-2$, it can be proved that $j^{\prime}(s)$ has only one positive

root. Since any solution $u$ is such that $\|u\|_{\infty} \leq \lambda^{1 /(p-1)}$, it is enough to show that $j\left(\lambda^{1 /(p-1)}\right)=\lambda(p-1)>0$, which is true.

Finally, taking $\phi_{1}$ as test function we deduce that for $\lambda \leq \lambda_{1}$ does not exist any solution.

With respect to items (3) and (4), the uniqueness follows from Theorem 4.3. Moreover as in the proof of Theorem 1.1, using that $\lambda u-u^{p} \leq \lambda u$ we obtain that $\lambda_{1} \leq(1-k) \lambda$ for $k<1$ and $\lambda_{1} \leq(1-c) \lambda$ for every $c<1$ if $k \geq 1$. This implies that for $k \geq 1$ problem (1.1) does not admit positive solution.

Finally item (5) follows from Corollary 2.8.

\section{Acknowledgements.}

Research supported by MICINN Ministerio de Ciencia e Innovación, Spain under grants MTM2012-31799 (JC and PMA) and MTM2012-31304 (AS) and Junta de Andalucía FQM-116 (PMA), FQM-194 (JC) and FQM-131 $(\mathrm{AS})$.

\section{References}

[1] B. Abdellaoui, I. Peral and A. Primo, Elliptic problems with a Hardy potential and critical growth in the gradient: non-resonance and blowup results, J. Differential Equations 239 (2007), no. 2, 386-416. 
[2] D. Arcoya, J. Carmona, T. Leonori, P. J. Martínez-Aparicio, L. Orsina and F. Petitta, Existence and nonexistence of solutions for singular quadratic quasilinear equations, J. Differential Equations 246 (2009), no. $10,4006-4042$.

[3] D. Arcoya, J. Carmona, P. J. Martínez-Aparicio, Bifurcation for quasilinear elliptic singular BVP, Comm. Partial Differential Equations 36 (2011), no. 4, 670-692.

[4] D. Arcoya, P. J. Martínez-Aparicio, Quasilinear equations with natural growth, Rev. Mat. Iberoam. 24 (2008), no. 2, 597-616.

[5] D. Arcoya and S. Segura de León, Uniqueness of solutions for some elliptic equations with a quadratic gradient term, ESAIM Control Optim. Calc. Var. 16 (2010), no. 2, 327-336.

[6] L. Boccardo, Dirichlet problems with singular and quadratic gradient lower order terms, ESAIM: Control, Optimization and the Calculus of Variations, 14 (2008) 411-426.

[7] L. Boccardo, F. Murat, Almost everywhere convergence of the gradients of solutions to elliptic and parabolic equations, Nonlinear Anal. 19 (1992) 581-597.

[8] L. Boccardo, F. Murat and J. P. Puel, Existence de solutions non bornes pour certaines quations quasi-linaires, Portugal. Math. 41 (1982), no. 1-4, 507-534 (1984).

[9] L. Boccardo, F. Murat and J. P. Puel, Résultats d'existence pour certains problèmes elliptiques quasilinéaires, Ann. Scuola Norm. Sup. Pisa Cl. Sci. (4) 11 (1984), no. 2, 213-235.

[10] L. Boccardo, L. Orsina, M. A. Porzio, Existence results for quasilinear elliptic and parabolic problems with quadratic gradient terms and sources, Adv. Calc. Var., 4 (2011), no. 4, 397-419.

[11] D. Giachetti and F. Murat, An elliptic problem with a lower order term having singular behaviour, Boll. Unione Mat. Ital. (9) 2 (2009), no. 2, 349-370. 
[12] O. Ladyzenskaya and N. Uralt'seva, Linear and quasilinear elliptic equations; Translated by Scripta Technica. - New York, Academic Press, 1968.

[13] J. Leray and J.L. Lions, Quelques résultats de Višik sur les problèmes elliptiques non linéaires par les méthodes de Minty-Browder, Bull. Soc. Math. France, 93 (1965), 97-107.

[14] L. Orsina, J. P. Puel, Positive solutions for a class of nonlinear elliptic problems involving quasilinear and semilinear terms, Comm. Partial Differential Equations, 26 (2001), no. 9-10, 1665-1689.

[15] D. Ruiz, A. Suárez, Existence and uniqueness of positive solution of a logistic equation with nonlinear gradient term, Proc. Roy. Soc. Edinburgh Sect. A, 137 (2007), no. 3, 555-566.

[16] G. Stampacchia, Le problème de Dirichlet pour les équations elliptiques du second ordre à coefficients discontinus, Ann. Inst. Fourier (Grenoble), 15 (1965), 189-258.

[17] W. Zhou, X. Wei and X. Qin, Nonexistence of solutions for singular elliptic equations with a quadratic gradient term, Nonlinear Analysis, 75 (2012), 5845-5850. 